\title{
Selective enhancement of emotional, but not motor, learning in monoamine oxidase A-deficient mice
}

(amygdala/norepinephrine/serotonin/cerebellum/mutant mice)

\author{
Jeansok J. Kim*†, Jean C. Shih $\$$, Kevin Chen $\ddagger$, Lu Chen*, Shaowen Bao*, Stephen Maren§, \\ Stephan G. Anagnostaras§, Michael S. Fanselow\$, Edward De Maeyer ${ }^{\top}$, Isabelle Seif ${ }^{\Uparrow}$, \\ AND RICHARD F. THOMPSON*
}

* Neuroscience Program, University of Southern California, Los Angeles, CA 90089-2520; \#Department of Molecular Pharmacology and Toxicology, University of Southern California, Los Angeles, CA 90033; §Department of Psychology, University of California, Los Angeles, CA 90024-1563; and ${ }^{\Uparrow}$ Centre National de la Recherche Scientifique, Unite Mixte de Recherche 177, Institut Curie, 91405 Orsay, France

Contributed by Richard F. Thompson, March 31, 1997

\begin{abstract}
Mice deficient in monoamine oxidase A (MAOA), an enzyme that metabolizes monoamines such as norepinephrine and serotonin, have elevated norepinephrine and serotonin levels in the frontal cortex, hippocampus, and cerebellum, compared with normal wild-type mice. Since monoamines in these areas are critically involved in a variety of behaviors, we examined learning and memory (using emotional and motor tasks) in MAOA mutant mice. The MAOAdeficient mice exhibited significantly enhanced classical fear conditioning (freezing to both tone and contextual stimuli) and step-down inhibitory avoidance learning. In contrast, eyeblink conditioning was normal in these mutant mice. The female MAOA-deficient mice also displayed normal speciestypical maternal behaviors (nesting, nursing, and pup retrieval). These results suggest that chronic elevations of monoamines, due to a deletion of the gene encoding MAOA, lead to selective alterations in emotional behavior.
\end{abstract}

Monoamines, which include catecholamines such as dopamine, norepinephrine (NE), and epinephrine, and indolamines such as serotonin (5-HT), are critically involved in behaviors ranging from sleep to ingestion (1), and aberrant levels of monoamines are correlated with mental dysfunctions (e.g., schizophrenia and depression) and neurological disorders (e.g., Alzheimer and Parkinson diseases) (2-5). The major monoaminergic systems emanate from several brainstem nuclei (e.g., NE in the locus coeruleus, dopamine in the substantia nigra, and 5-HT in the raphe nucleus) and they exert their influences by sending both ascending and descending projections to various regions of the brain and the spinal cord (6).

Recently, mutant mice lacking monoamine oxidase A (MAOA), one of two mitochondrial membrane-bound MAO isoenzymes (the other being MAOB) which degrades monoamines to inert metabolites (7), have been shown to exhibit enhanced aggression (e.g., in the resident-intruder test) and cytoarchitectural changes in their somatosensory cortex (specifically the absence of barrelfield) $(8,9)$. Similarly to what is observed in normal mice using drugs that block MAOA (10), in MAOA-deficient brains, NE and 5-HT levels were significantly elevated (8). The enhanced aggressive behavior exhibited by the MAOA mutant mice is consistent with the impulsive aggression reported in men from a Dutch family with a complete MAOA deficiency, due to a point mutation in the gene encoding MAOA (11). In addition to abnormal aggres-

The publication costs of this article were defrayed in part by page charge payment. This article must therefore be hereby marked "advertisement" in accordance with 18 U.S.C. $\$ 1734$ solely to indicate this fact.

Copyright (C) 1997 by The National Academy of Sciences of the USA 0027-8424/97/945929-5\$2.00/0

PNAS is available online at http://www.pnas.org sive behavior, these men are also afflicted with borderline mental retardation.

To test whether MAOA-deficient mice also manifest cognitive deficits, we examined learning and memory performance in these animals by using both emotional (classical fear conditioning and step-down inhibitory avoidance) and motor (classical eyeblink conditioning) tasks. Fear conditioning occurs when arbitrary stimuli such as tones, lights, or distinctive environments are paired with aversive unconditioned stimuli (US) such as footshock. Through association formation, arbitrary stimuli become conditioned stimuli (CS) that are capable of eliciting fear responses. In the step-down inhibitory avoidance, the animal must remember that stepping off a safe platform resulted in a footshock. Eyeblink conditioning takes place when a discrete CS (usually tone or light) is paired with a discrete US (usually airpuff or periorbital shock) directed at the eye. The animal initially blinks only to the US; this reflexive eyeblink to the US is known as the unconditioned response. Over the course of training, the animal gradually blinks to the CS; this learned eyeblink to the CS is known as the conditioned response. In addition to learning tasks, species-typical maternal behaviors such as nesting, nursing, and pup retrieval were examined in postparturient MAOAdeficient mice. Finally, monoamine levels were determined in the hippocampus, the frontal cortex, and the cerebellum, structures implicated in some forms of learning and memory (12-14).

\section{MATERIALS AND METHODS}

Subjects. Thirteen MAOA-deficient (4 males and 9 females) and nine wild-type control (5 males and 4 females) mice of $\mathrm{C} 3 \mathrm{H} / \mathrm{HeJ}$ genetic background $(\approx 4$ months old) underwent two different types of emotional learning tasks (fear conditioning and step-down avoidance) followed by eyeblink (motor) conditioning. The animals had free access to food and water and all behavioral tests were carried out during the light phase of the light/dark cycle. At the end of the experiment, the genotypes of the mice were reconfirmed by PCR analysis of DNA prepared from tails (8).

Fear Conditioning. For fear conditioning, animals were housed (groups of 1-3 per cage) in the University of California, Los Angeles, Psychology Department vivarium for 2 weeks of adaptation (a 14-hr light/10-hr dark cycle, lights on at 7 a.m.) prior to the start of training. On day 1 of fear conditioning, each mouse was placed in one of four identical experimental

Abbreviations: MAOA, monoamine oxidase A; NE, norepinephrine; 5-HT, serotonin; US, unconditioned stimuli; CS, conditioned stimuli; EMG, electromyograph.

†To whom reprint requests should be addressed at: Department of Psychology, 2 Hillhouse Avenue, Yale University, New Haven, CT 06520-8205. e-mail: jeansok.kim@yale.edu. 
chambers $(28 \times 21 \times 22 \mathrm{~cm}$; Lafayette Instruments, North Lafayette, IN) with the floor consisting of 24 stainless steel rods (1-mm diameter) spaced $0.5 \mathrm{~cm}$ apart (center-to-center). After $3 \mathrm{~min}$ in the chamber, the mice received three tonefootshock pairings (tone: $10 \mathrm{sec}, 85 \mathrm{~dB}, 2 \mathrm{kHz}$; footshock: $1 \mathrm{sec}$, $0.5 \mathrm{~mA}$; intertrial interval $1 \mathrm{~min}$ apart). One minute after the final footshock, the mice were returned to their home cages. Each chamber was wiped with $5 \%$ ammonium hydroxide solution before training. On day 2, fear conditioning to the context was assessed by placing the mice back in the conditioning chamber for an 8-min test, in the absence of any tone and footshock. On day 3, fear conditioning to the tone was measured in observation chambers that were different from those used during conditioning (15). After $2 \mathrm{~min}$ in the new chamber, the conditioned tone was presented for a 6-min test. Fear conditioning was assessed during training (day 1), context test (day 2), and tone test (day 3) by measuring the freezing response. Freezing is defined as the absence of all visible movements of the body and vibrissae aside from movement necessitated by respiration. An observer who was blind to the experimental conditions scored each mouse for freezing every $8 \mathrm{sec}$. The freezing data were transformed to a percentage of total observations, a probability estimate that is amenable to analysis of variance.

Step-Down Avoidance. Promptly following fear conditioning, the animals were transported and individually housed in the University of Southern California, HEDCO Neuroscience Building vivarium (a 12-hr light/12-hr dark cycle, lights on at 7 a.m.). After 2 weeks of adaptation, the mice underwent step-down avoidance training. In this task, each mouse was placed on a $9-\mathrm{cm}$ diameter platform that was elevated $1.5 \mathrm{~cm}$ from the grid floor of the experimental chamber $(21 \times 23 \times$ $26 \mathrm{~cm}$; Ralph Gerbrands, Arlington, MA). Immediately upon stepping down (all four paws on the grid floor), the animals received a single footshock $(1 \mathrm{sec}, 0.5 \mathrm{~mA})$. After the footshock, the animals were returned to their home cage. The mice were placed back on the platform 1,2, and 7 days following the footshock and step-down latencies were measured; the maximum time allowed on the platform was $3 \mathrm{~min}$.

Eyeblink Conditioning. One week after step-down avoidance testing, animals underwent eyeblink conditioning using a previously described method in mice (16). In brief, under ketamine $(80 \mathrm{mg} / \mathrm{kg}$, i.p.) and xylazine $(20 \mathrm{mg} / \mathrm{kg}$, i.p. $)$ anesthesia, mice were implanted subcutaneously with four Tefloncoated stainless steel wires (0.003-inch bare, 0.0045-inch coated; A-M Systems, Everett, WA) to the left upper eyelid. The tips of the wires were exposed, and two of the wires were used to record differential electromyograph (EMG) from obicularis oculi, and the remaining two wires were used to deliver periorbital shock. A four-pin strip connector to which the wires were soldered was cemented to the skull of the animal with dental acrylic. Two to 3 days after the surgery, each mouse was placed in one of four identical cylindrical Plexiglas containers (3.75-inch diameter), which was placed inside a sound- and light-attenuating chamber. The strip connector was attached to the mating plug of a commutator with channels to relay EMG signals and to deliver the shock. The daily training consisted of 100 trials grouped in 10 blocks. Each block included one CS alone, one US alone, and eight CS-US paired trials. The CS and US were a $352-\mathrm{msec}$ tone $(1000 \mathrm{~Hz}, 80 \mathrm{~dB})$ and a coterminating $100-\mathrm{msec}$ shock $(100-\mathrm{Hz}$ biphasic square pulses), respectively, with a randomized intertrial interval between 20 and $40 \mathrm{sec}($ mean $=30 \mathrm{sec})$. The US intensity used was the minimal voltage required to elicit an eyeblink/head turn response (unconditioned response), and the US intensity was adjusted daily for each animal (ranging from 3 to $60 \mathrm{~V}$ ). After 7 days of paired training, all animals received 4 days of CS-alone extinction trials. The EMG signal was amplified in the band of $300-5,000 \mathrm{~Hz}$ and sent to a window discriminator where the number of pulses above the noise envelop was sampled and stored by a computer. The discriminated EMG activity was then analyzed trial by trial using an EMG unit analysis program that made statistical comparisons between pre-CS EMG activity and CS EMG activity (16).

Maternal Behavior. Experimentally naive, postparturient (first litter) MAOA $(n=4)$ and wild-type mice $(n=4)$ (between 3 and 4 months old) were examined for nesting, nursing, and pup retrieval behaviors. The nesting behavior was scored as 0 for no nesting, 1 for incomplete nesting (no enclosing walls), and 2 for complete nesting (enclosing walls). The nursing behavior, defined as crouching and providing milk to the pups, was assigned 0 for the absence and 1 for the presence of the behavior. The pup retrieval behavior was assessed by the average time it took the mother to retrieve displaced pups (the total time required to retrieve all pups/ number of pups); the pups were placed on the opposite end of the cage from the nesting area. These maternal behaviors were observed once a day for 5 consecutive days after the pups were born.

Determination of Dopamine, NE, and 5-HT Levels in the Brain Tissue. The frontal cortex, the hippocampus, and the cerebellum were rapidly dissected from the brains of experimentally naive normal and mutant mice and frozen in liquid nitrogen until prepared for HPLC analysis (as described in ref. 17). Each brain region was homogenized in a solution containing $0.1 \mathrm{M}$ trichloroacetic acid, $10 \mathrm{mM}$ sodium acetate, and $0.1 \mathrm{mM}$ EDTA ( $\mathrm{pH} 3.75$ ); $1 \mu \mathrm{M}$ isoproterenol was used as an internal standard. The homogenates were sonicated for $5 \mathrm{sec}$ (Fisher Sonic Dismembrator, model 300; probe sonicator at setting 60), centrifuged, and the supernatants were divided into aliquots for HPLC analysis. The protein concentrations were determined using the pellet with the method of Lowry (18) with bovine serum albumin as standard.

HPLC analysis was performed using 5-HT and NE (Sigma) as standards. The mobile phase was the same as the homogenization buffer (excluding the isoproterenol) with $15 \%$ methanol for detection of 5-HT. NE was quantified separately using $5 \%$ methanol in the trichloroacetic acid mobile phase solution. The mobil phases were filtered and deaerated and the pump speed (Shimadzu LC-6A liquid chromatograph) was $1.5 \mathrm{ml}$ per minute. The reverse-phase column used was a Rexchrom S50100-ODS $C_{18}$ column with a length of $25 \mathrm{~cm}$ and an internal diameter of $4.6 \mathrm{~mm}$ (Regis, Morton Grove, IL). The compounds were measured at $+0.7 \mathrm{~V}$ using a Shimadzu L-ECD-6A electrochemical detector. Standard curves were determined for 5-HT and NE and the amount of the different compounds in each sample was determined by comparison with the standard curve taking into account a correction factor based upon the amount of the internal standard isoproterenol in the sample.

\section{RESULTS}

The freezing, step-down avoidance latency, and eyeblinkconditioned response data were pooled across sex, since there were no differences between males and females, in both MAOA mutant and wild-type mice.

As shown in Fig. 1, fear conditioning (percent freezing) was significantly elevated in the MAOA-deficient mice, in comparison to the wild-type mice, during training [immediate postshock freezing during minutes 4,5 , and $6 ; F(1,21)=$ 19.97; $P<0.01]$, context test $[F(1,21)=5.86 ; P<0.05]$, and tone test $[F(1,21)=17.32 ; P<0.01]$. Prior to the footshock (minutes 1, 2, and 3), neither the MAOA or wild-type mice displayed any freezing behavior.

The MAOA-deficient mice also demonstrated significantly enhanced step-down avoidance latencies on day $1[F(1,21)=$ $7.37 ; P<0.05]$, day $2[F(1,21)=27.63 ; P<0.01]$, and day $7[F(1,21)=24.26 ; P<0.01]$ of training-test intervals when 


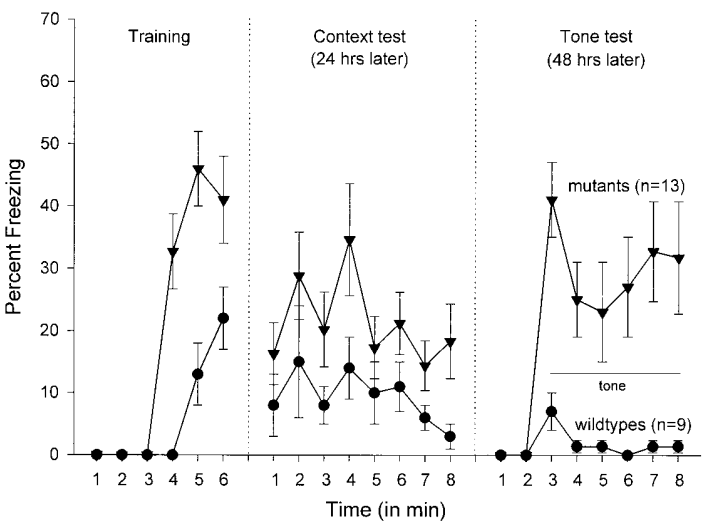

FIG. 1. Mean $( \pm$ SEM $)$ freezing level during training, context test, and tone test. In the training, 1, 2, and 3 denote $3 \mathrm{~min}$ of baseline (pretone footshock) and 4, 5, and 6 represent three posttone footshock intervals. The context test was for $8 \mathrm{~min}$. In the tone test, 1 and 2 signify 2 min of baseline (in a new context) prior to 6 min of tone onset. Freezing scores are expressed as the percentage of total observation.

compared with the wild types (Fig. 2). Although there was a trend of MAOA-deficient mice having a longer latency than the wild types to step-down from the platform prior to the shock (baseline), this difference was not statistically significant $[F(1,21)=3.82 ; P>0.05]$. However, there was no reliable group $\mathrm{x}$ training-test interval interaction with the baseline $[F(3,66)=1.52 ; P>0.05]$.

In contrast to fear conditioning and step-down inhibitory avoidance, there was no statistically reliable difference between the MAOA mutant and the wild-type mice in eyeblink conditioning $(P>0.05)$ (Fig. $3 A)$. Both groups showed gradual acquisition of eyeblink conditioned responses over 7 days of CS-US paired training, and demonstrated extinction during the 4 days of CS-alone trials. However, the MAOA mutants appear to be less sensitive to the periorbital shock US since the minimal US intensity (voltage) required to elicit an eyeblink/ head turn response was significantly higher in the MAOAdeficient mice than in normal mice across the 7 days of training [group $\times$ day interaction; $F(6,132)=4.72 ; P<0.01$ ] (Fig. $3 B)$.

There were no noticeable differences in the species-typical maternal behavior exhibited by postparturient MAOAdeficient and wild-type mice (Table 1). Both groups demonstrated comparable nesting, nursing, and pup-retrieval behaviors to their first litter.

When compared with the wild types, the MAOA mutants showed significantly elevated levels of NE and 5-HT in the hippocampus, the frontal cortex, and the cerebellum (Table 2).

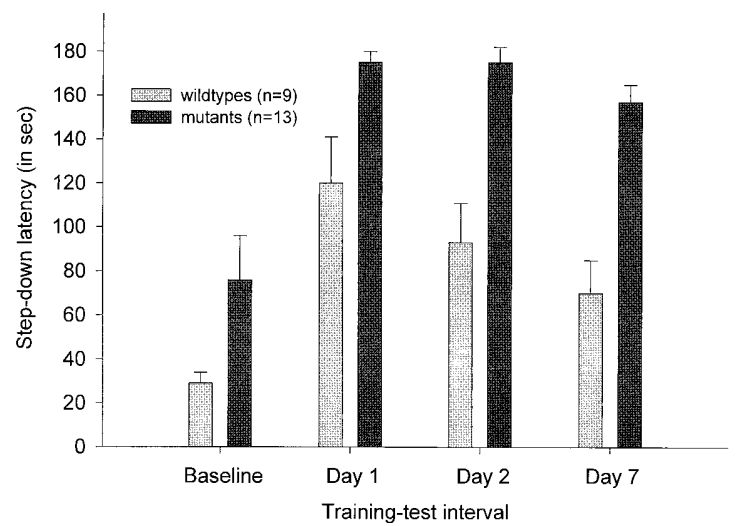

FIG. 2. Mean ( \pm SEM) latency to step down from the platform prior to the footshock (Baseline), 1 day after training (Day 1), 2 days after training (Day 2), and 7 days after training (Day 7).
A
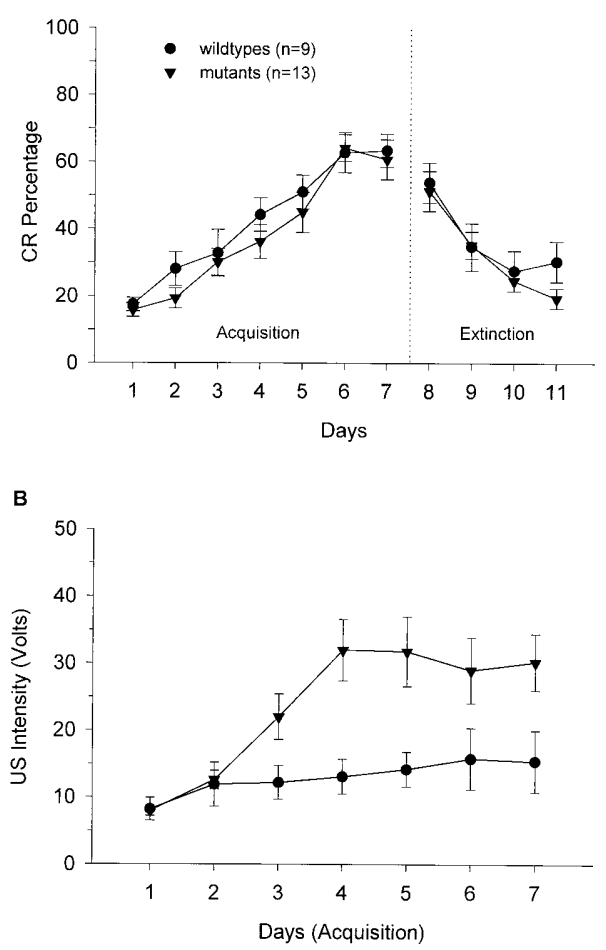

FIG. 3. (A) Mean ( \pm SEM) percentage of conditioned responses exhibited by MAOA-deficient and wild-type mice during 7 days of acquisition and 4 days of extinction. $(B)$ Mean ( \pm SEM) periorbital shock (US) intensity used for eyeblink conditioning in MAOA and wild-type mice during 7 days of acquisition. The US intensity, the minimal voltage required to elicit an eyeblink/head turn response, was adjusted daily for each animal.

\section{DISCUSSION}

We found that mice deficient in MAOA enzymatic activity have elevated levels of monoamines (specifically $\mathrm{NE}$ and 5-HT) in the brain and display an enhancement in emotional (fear) learning. In the fear conditioning task, neither the MAOA mutants nor the wild types exhibited any freezing behavior prior to the first footshock. However, the MAOAdeficient mice exhibited heightened freezing responses during training (immediate postshock periods), context test ( $24 \mathrm{hr}$ later), and tone test ( $48 \mathrm{hr}$ later) in comparison to normal mice. Similarly, the MAOA-deficient mice manifested longer latencies to step down from a safe platform than the normal mice in the inhibitory avoidance learning. Thus, it appears that fear learning is generally enhanced in these mutants.

The enhancement of fear learning observed in MAOAdeficient mice may be due to elevated catecholamine levels, because injections of catecholamines into the brains of mice enhances fear memory formation (19), whereas drugs that lower the levels of catecholamines impair it (20). Since the amygdala is critically involved in both fear conditioning and inhibitory avoidance learning (e.g., refs. 21-24), it is possible

Table 1. Maternal behaviors of wild-type and MAOA mutant mice

\begin{tabular}{lccc}
\hline Genotype & Nesting & Nursing & Pup retrieval \\
\hline Wild type & $1.35 \pm 0.19$ & $0.8 \pm 0.08$ & $12.7 \pm 2.69$ \\
MAOA & $1.50 \pm 0.19$ & $0.19 \pm 0.10$ & $14.4 \pm 2.94$ \\
\hline
\end{tabular}

Values are shown by mean \pm SEM. Nesting: 0 , no nesting; 1 , incomplete nesting; and 2, complete nesting. Nursing: 0 , no nursing; 1 , nursing. Pup retrieval, the average time (seconds) it took the mother to retrieve displaced pups. Wild-type mice $(n=4)$; MAOA mice $(n=$ 4). 
Table 2. Concentrations of neurotransmitters in various brain regions of the wild-type and MAOA mutant mice

\begin{tabular}{ccrc}
\hline Brain region & Neurotransmitters & \multicolumn{1}{c}{ Wild types } & MAOA mutants \\
\hline Cerebellum & 5 -HT & $17.12 \pm 3.06$ & $45.39 \pm 3.33^{* *}$ \\
& NE & $10.29 \pm 2.08$ & $16.88 \pm 4.53^{*}$ \\
Frontal cortex & $5-\mathrm{HT}$ & $17.73 \pm 3.10$ & $38.24 \pm 6.81^{*}$ \\
& $\mathrm{NE}$ & $8.22 \pm 0.97$ & $16.62 \pm 2.06^{* *}$ \\
Hippocampus & $5-\mathrm{HT}$ & $25.02 \pm 13.28$ & $70.43 \pm 8.27^{* *}$ \\
& $\mathrm{NE}$ & $12.08 \pm 3.47$ & $19.42 \pm 4.01^{*}$ \\
\hline
\end{tabular}

Concentrations are in $\mathrm{pmol} / \mathrm{mg}$ protein. Values are shown by mean \pm SD. For 5 -HT, $n=4$ in wild-type and $n=4$ in MAOA mutant mice. For NE, $n=4$ in wild types and $n=5$ in MAOA mutants. $*, P<0.05$; **, $P<0.01$ ( $t$ test $)$

that the alteration of catecholamine levels in the amygdala is responsible for the enhancement of fear learning observed in the MAOA mutant mice. Other pharmacological studies indicate that treatments that increase the noradrenergic transmission in the amygdala (e.g., infusions of $\mathrm{NE}$ into the amygdala) enhance fear learning, whereas treatments that decrease noradrenergic transmission impair fear learning (e.g., infusions of noradrenergic antagonists) $(25,26)$. It is conceivable then that the enhancement of emotional learning exhibited by the MAOA-deficient mice is due to the elevation of NE in the amygdala. Although the NE level was not examined specifically in the amygdala, since the locus coeruleus is known to innervate the amygdala (1), the absence of MAOA activity would most likely result in the elevation of NE level in the amygdala. Consistent with this view, the NE level was elevated in other brain structures innervated by the locus coeruleus, such as the frontal cortex, the hippocampus, and the cerebellum.

The effect on emotional learning appears to be reasonably selective, since maternal behavior and eyeblink conditioning were not affected. For instance, there were no observable differences in the species-typical maternal behavior displayed by postparturient MAOA-deficient and wild-type mice. Both groups exhibited comparable nesting, nursing, and pupretrieval behaviors to their first litter. The lack of effect on eyeblink conditioning was surprising given that monoaminergic afferents constitute one of the three major afferent systems in the cerebellum (the others are the mossy fiber and climbing fiber afferents) (27). The monoaminergic inputs include welldefined noradrenergic and serotonergic afferents from the locus coeruleus and raphe nucleus, respectively. The functional roles of these monoaminergic afferents are not clear, but there is evidence that they modulate synaptic transmission in Purkinje cells and other cerebellar cortical neurons. For instance, iontophoretic application of NE to Purkinje cells results in an enhancement of both excitatory and inhibitory responses of Purkinje cells, both to mossy fiber and climbing fiber inputs (28). In eyeblink conditioning, there is evidence indicating that the mossy fibers and the climbing fibers relay information about the CS and the US, respectively (14). Because MAOA-deficient mice showed a normal rate of acquisition of eyeblink conditioning, either monoaminergic systems are not involved in eyeblink conditioning, the monoamine levels were not sufficiently elevated to influence eyeblink conditioning, or the elevated monoamine levels resulted in some form of developmental compensation. Thus, the lack of an eyeblink conditioning effect in MAOA-deficient mice cannot rule out the involvement of monoamine systems in the normal eyeblink conditioning situation.

Interestingly, there was a significant difference in the minimal periorbital shock US intensity required to elicit an eyeblink/head turn response between MAOA mutant and wild-type mice. Although they initially started out at comparable intensities, the MAOA-deficient mice needed increasingly higher voltages than the wild types to elicit the same unconditioned response over the course of training sessions. This finding suggests that MAOA mutants may have an elevated pain threshold. It is conceivable that the alteration in pain sensitivity is due to the elevated 5-HT level. For example, decreasing brain 5-HT increases sensitivity to electric shock in rats, whereas increasing brain 5-HT decreases pain sensitivity (29). It is also possible that the MAOA mutants exhibit stronger conditioned analgesia (due to enhance fear conditioning associated with periorbital shock US) and therefore subsequently require stronger US intensities. Conditioned fear is known to decrease pain sensitivity via activating an endogenous analgesic system (30). This may explain why MAOAdeficient and wild-type mice did not differ in US intensities prior to undergoing eyeblink conditioning. Thus, the MAOA mutants might have demonstrated impaired eyeblink conditioning if a constant US intensity had been used (rather than variable US intensities adjusted to each animal). Regardless, the enhanced fear learning (i.e., conditioned freezing and step-down avoidance) observed in the MAOA mutant mice is unlikely to be due to an increase in pain sensitivity since they appear to be less sensitive to (periorbital) electric shock.

As mentioned in the Introduction, the men of a Dutch family with deficient MAOA activity manifest impulsive aggression as well as a borderline mental retardation (11). The MAOA mutant mice similarly exhibit enhanced aggressive behavior (8), and as indicated by the present results, also show a selective enhancement in the performance of emotional (fear), but not motor (eyeblink conditioning), tasks. [It is not known if fear conditioning is modified in humans with MAOA deficiency. It is also possible (but not yet tested) that more complex behavioral tasks like spatial memory may be impaired in these mice.]. Although the enhanced performance in fear tasks shown by MAOA-deficient mice might be due to an alteration of the fear memory process per se as mentioned above (e.g., the elevated NE levels modulating memory consolidation process), it cannot be ruled out that there may be a general alteration of emotional reactivity to threatening (learned or unlearned) stimuli in the MAOA-deficient mice. Since MAOA mutants lack barrelfields in their somatosensory cortex $(8,9)$, it is possible that their exploratory behavior is altered to favor freezing and step-down avoidance. Indeed, there was a trend toward MAOA-deficient mice taking a longer latency to step-down than the wild types prior to experiencing the footshock. However, the preshock baseline latency did not correlate with postshock test latencies, indicating that a longer latency to step-down prior to the footshock does not necessarily lead to enhanced step-down inhibitory avoidance learning. Likewise, in the fear conditioning experiment, the MAOA mutants exhibited less cage crossover behavior than the wild types during the 3-min preshock baseline (data not shown). Again, the crossover behavior and the conditioned freezing response did not correlate, suggesting that the limited crossover behavior is not responsible for the augmented conditioned freezing. Pharmacological manipulations, such as infusions of various monoaminergic drugs (e.g., $\beta$-adrenergic receptor antagonist propranolol) into various brain structures (e.g., the amygdala) prior to training and testing, may provide the means to separate this learning and performance issue in MAOA-deficient mice.

We thank Michael Davis and James L. McGaugh for comments on this manuscript and Mi Jeong Shin for assistance. This work was supported by grants from the National Institute of Mental Health (1F32MN10521-01 BNR) to J.J.K., from the National Institute of Mental Health (R37 MH39085, MERIT Award, K05 MH00795 RSAward, ROMH37020) and Welin Professorship Award to J.C.S., and from the National Science Foundation (BNS-8718300), the National Institutes of Health (AG05142), and Sankyo to R.F.T. 
1. Feldman, R. S. \& Quenzer, L. F. (1984) Fundamentals of Neuropsychopharmacology (Sinauer, Sunderland, MA).

2. Snyder, S. H. (1974) Science 184, 1243-1253.

3. Palmer, A. M. \& DeKosky, S. T. (1993) J. Neural Transm. Gen. Sect. 91, 135-159.

4. Schildkraut, J. J. (1965) Am. J. Psychiatry 122, 509-522.

5. Hornykiewicz, O. (1974) Life Sci. 15, 1249-1259.

6. Elliott, G. R., Edelman, A. M., Renson, J. F. \& Berger, P. A (1977) in Psychopharmacology: From Theory to Practice, eds. Barchas, J. D., Berger, P. A., Cicaranello, R. D. \& Elliott, G. R. (Oxford Univ. Press, New York), pp. 33-50.

7. Shih, J. C. (1990) Neuropsychopharmacology 4, 1-7.

8. Cases, O., Seif, I., Grimsby, J., Gaspar, P., Chen, K., Pournin, S., Muller, U., Aguet, M., Babinet, C., Shih, J. C. \& De Maeyer, E. (1995) Science 268, 1763-1766.

9. Cases, O., Vitalis, T., Seif, I., De Maeyer, E., Sotelo, C. \& Gaspar, P. (1996) Neuron 16, 297-307.

10. Freeman, H. (1993) Lancet 342, 1528-1532.

11. Brunner, H. G., Nelen, M., Breakefield, X. O., Ropers, H. H. \& van Oost, B. A. (1993) Science 262, 578-580.

12. Goldman-Rakic, P. S. (1992) Sci. Am. 267, 110-117.

13. Squire, L. R. \& Zola-Morgan, S. (1991) Science 253, 1380-1386.

14. Thompson, R. F. (1986) Science 233, 941-947.

15. Kim, J. J. \& Fanselow, M. S. (1992) Science 256, 675-677.

16. Chen, L., Bao, S., Lockard, J. M., Kim, J. J. \& Thompson, R. F. (1996) J. Neurosci. 16, 2829-2838.
17. Kalivas, P. W. (1985) J. Pharmacol. Exp. Ther. 235, 544-550.

18. Lowry, O. H., Rosebrough, N. J., Farr, A. L. \& Randall, R. J. (1951) J. Biol. Chem. 193, 265-275.

19. Haycock, J. W., van Buskirk, R. \& McGaugh, J. L. (1977) Behav. Biol. 20, 281-310.

20. Stein, L., Belluzzi, J. D. \& Wise, C. D. (1975) Brain Res. 84, 329-335.

21. Davis, M. (1992) Annu. Rev. Neurosci. 15, 353-375.

22. Lavond, D. G., Kim, J. J. \& Thompson, R. F. (1993) Annu. Rev. Psychol. 44, 317-342.

23. LeDoux, J. E. (1994) Sci. Am. 270, 50-57.

24. McGaugh, J. L. (1989) Annu. Rev. Neurosci. 12, 255-287.

25. McGaugh, J. L., Introini-Collison, I. B., Nagahara, A. H. \& Cahill, L. (1990) Neurosci. Biobehav. Rev. 14, 425-431.

26. Liang, K. C., McGaugh, J. L. \& Yao, H. Y. (1990) Brain Res. 508, 225-233.

27. Ito, M. (1984) The Cerebellum and Neural Control (Raven, New York).

28. Freedman, R., Hoffer, B. J., Woodward, D. J. \& Puro, D. (1977) Exp. Neurol. 55, 269-288.

29. Seiden, L. S. \& Dykstra, L. A. (1977) Psychopharmacology: A Biochemical and Behavioral Approach (Van Nostrand Reinhold, New York).

30. Fanselow, M. S. (1984) Trends Neurosci. 7, 460-462. 